\title{
Micro/nano processing of natural silk fibers with near-field enhanced ultrafast laser
}

\author{
Ming Qiao ${ }^{1 \dagger}$, Huimin Wang ${ }^{2 \dagger}$, Haojie $\mathrm{Lu}^{2}$, Shuo Li ${ }^{2}$, Jianfeng Yan ${ }^{1^{*}}$, Liangti Qu ${ }^{1}$, Yingying Zhang ${ }^{2^{*}}$, \\ Lan Jiang ${ }^{3}$ and Yongfeng Lu ${ }^{4}$
}

\begin{abstract}
Silkworm silk fiber is an attractive material owing to its remarkable mechanical characteristics, excellent optical properties, and good biocompatibility and biodegradability. However, nano-processing of the silk fiber is still a challenge limiting its applications in nanoengineering and related fields. Herein, we report localized near-field enhancement-assisted ablation with an ultrafast laser to break this bottleneck. Localized processing of silk fiber, including nano-holing, nano-grooving, and cutting could retain the key molecular structure building blocks and the pristine functionality of the silk fiber. An extremely narrow nanohole with a width of $\sim 64 \mathrm{~nm}$ was successfully achieved. The processed silk fiber can be used to transfer micro/nanoparticles and drugs, showing potential for biomedical engineering. The processing strategy developed in this study can also be extended to other materials, paving a new way for fabricating functional nanostructures with precisely controlled size and morphology.
\end{abstract}

Keywords: ultrafast laser, silk fiber, laser ablation, nano-processing, drug delivery

\section{INTRODUCTION}

Silkworm silk fiber is an attractive biocompatible material that has been used by humans for thousands of years owing to its superior properties [1]. It shows promise as a kind of biological polymer material that can be applied in cutting-edge fields owing to its unique structures, remarkable mechanical characteristics, excellent optical properties, facile chemical functionalization, and good biocompatibility and biodegradability. Pioneering studies have been performed to develop applications of the silk fiber in various fields, such as functional fabrics, silk muscles, conductive fibers, and optical fibers [2-5]. Despite these advances, the interests in exploring the potential of silk fiber for nanoengineering and related applications are growing.

Chemical processing can transform silk fibers into transparent films or bulk structures. Besides, nanoimprinting, inkjet printing, multiphoton lithography, and template-assisted patterning of silk-fibroin films have been explored to fabricate nano-scale structures [6-9]. Various applications of silk-fibroin films, such as in implantable bioelectronics $[6,10,11]$, biomedical diagnosis $[12,13]$, organic photovoltaics devices $[14,15]$, and brainmachine interfaces [16,17], have been demonstrated. However, the structures of those silk films or bulk structures are different from that of the individual silkworm silk fiber, which results in loss of some function and strength [6-9]. Therefore, a non-invasive processing approach for individual silkworm silk fibers is desired. Compared with the methods mentioned above, ultrafast laser micro/nano fabrication is endowed with low collateral damage $[18,19]$, maskless capability $[20,21]$, excellent biocompatibility of fabrication process [21,22], high machining accuracy $[9,23,24]$, and versatile applicability to various materials $[19,25,26]$.

Herein, localized near-field enhancement-assisted ablation with a femtosecond laser was reported to process nano-scale structures on individual silk fiber. Two dif-

\footnotetext{
${ }^{1}$ Department of Mechanical Engineering, Tsinghua University, Beijing 100084, China

${ }^{2}$ Key Laboratory of Organic Optoelectronics and Molecular Engineering of the Ministry of Education, Department of Chemistry, Tsinghua University, Beijing 100084, China

${ }^{3}$ Laser Micro/Nano Fabrication Laboratory, School of Mechanical Engineering, Beijing Institute of Technology, Beijing 100081, China

${ }^{4}$ Department of Electrical and Computer Engineering, University of Nebraska-Lincoln, Lincoln, NE 68588-0511, USA

† These authors contributed equally to this work.

* Corresponding authors (emails: yanjianfeng@tsinghua.edu.cn (Yan J); yingyingzhang@tsinghua.edu.cn (Zhang Y))
} 
ferent modes, far-field laser ablation (Mode 1) and localized near-field enhancement-assisted ablation (Mode 2), were presented to distinguish the conventional ultrafast laser micro-nano processing strategy and our novel processing strategy. Different localized processings, including nano-holing, nano-grooving, and cutting, were demonstrated. Furthermore, we also transferred microparticles into the laser-ablated holes on the silk fiber and fabricated diverse patterns on commercially available silk fabrics. The novel processing approach is expected to be applied on a variety of materials for constructing functional nanostructures.

\section{EXPERIMENTAL SECTION}

\section{Preparation of degummed silkworm silk fibers}

The silkworm cocoons and commercially available silk fabrics were purchased from https://vasana.tmall.com/. The obtained cocoons were cut into pieces and boiled several times in an aqueous solution of $0.5 \%(w / w)$ $\mathrm{NaHCO}_{3}$ for $1 \mathrm{~h}$ and then rinsed thoroughly with deionized water. The collected degummed silks were dried at $60^{\circ} \mathrm{C}$ in an oven for $8 \mathrm{~h}$. Then, single degummed silk was placed on a piece of silicon slice with atomic level flatness.

\section{Femtosecond laser processing}

A femtosecond pulse laser (35 fs) with repetition frequency of $1-1000 \mathrm{~Hz}$ was used to process individual silk fiber and silk fabrics. A half-wave plate was used to control the laser polarization direction. A neutral density filter was used to regulate the pulse energy fluence, and a shutter was used to control the number of pulses deposited on the target position. The laser was focused on the surface of the sample by a microscope object $(\mathrm{NA}=$ 0.5 for the silk fiber and NA $=0.15$ for silk fabrics). To focus the laser pulses on the target position and pattern on the silk fabric, the sample was fixed on a computercontrolled positioning stage, and the processing process could be observed through a charge-coupled device (CCD) camera in situ. Furthermore, the processing time we used to process silk with different lasers was consistent (1 s).

\section{Simulation}

The optical intensity was calculated by the three-demensional (3D) Finite Difference Time Domain (FDTD) method. The simulation system was consistent with the experimental setup. An $800 \mathrm{~nm}$ laser with Gaussian distribution was linearly polarized along $x$ axis and focused on the surface of the silk fiber. The refractive indices used in the simulation was 1.58 for the silk fiber.

\section{Characterization}

The morphology and microstructure of the materials were characterized using a scanning electron microscope (SEM) (HITACHI SU8220). A universal testing machine (SHIMADZU AGS-X) was used to measure the mechanical properties of the native and processed silk fiber. Raman spectra were recorded using a Raman spectroscope (Horiba HR800, $532 \mathrm{~nm}, 1 \mu \mathrm{m}$ spot size). X-ray photoelectron spectroscopy (XPS) images were obtained using an XPS spectroscope (PHI Quantera SXM, ULVAC-PHI). Besides, we chose femtosecond laser patterned silk fabric for XPS characterization. The ultraviolet-visible-infrared absorbance and transmittance spectra of silk were measured by a spectrometer (Shimadzu UV2600).

\section{Transfer of polystyrene (PS) microsphere}

First, a PS microsphere suspension was prepared by dissolving $2 \mathrm{mg}$ of PS microspheres in $1 \mathrm{~mL}$ of water. A Ti probe was prepared through an electrochemical etching process under a potential of $50 \mathrm{~V}$ in $1 \mathrm{~mol} \mathrm{~L}^{-1} \mathrm{KOH}$. Then, the PS microsphere was attached to the tip of the Ti probe and placed in the hole created by the femtosecond laser.

\section{RESULTS AND DISCUSSION}

\section{Far-field laser ablation and localized near-field enhancement-assisted ablation}

The mechanism behind the interaction between ultrafast laser pulses and silk fibers was discussed first. Far-field laser ablation is conventionally used to process micronsized structures due to the optical diffraction limit. The optical near-field enhancement effect plays an important role in the precise processing of silk fibers. It is known that optical near-field enhancement can occur near metallic or dielectric nanostructures with dimensions smaller than the illumination wavelength [27]. The near-field enhancement with metallic nanostructures is induced by surface plasmon excitation [28]. Correspondingly, the near-field enhancement with dielectric nanostructures is related with lens-based near-field focusing or Mie resonances [29,30]. The natural silk fibers have wavy structures on the surface (Fig. S1), and the localized nearfield enhancement occurs on the silk fibers.

On ablation of the silk fibers with ultrafast laser pulses, three stages (Fig. 1) can be identified: (1) localized nearfield enhancement, (2) formation of plasma, and (3) ab- 


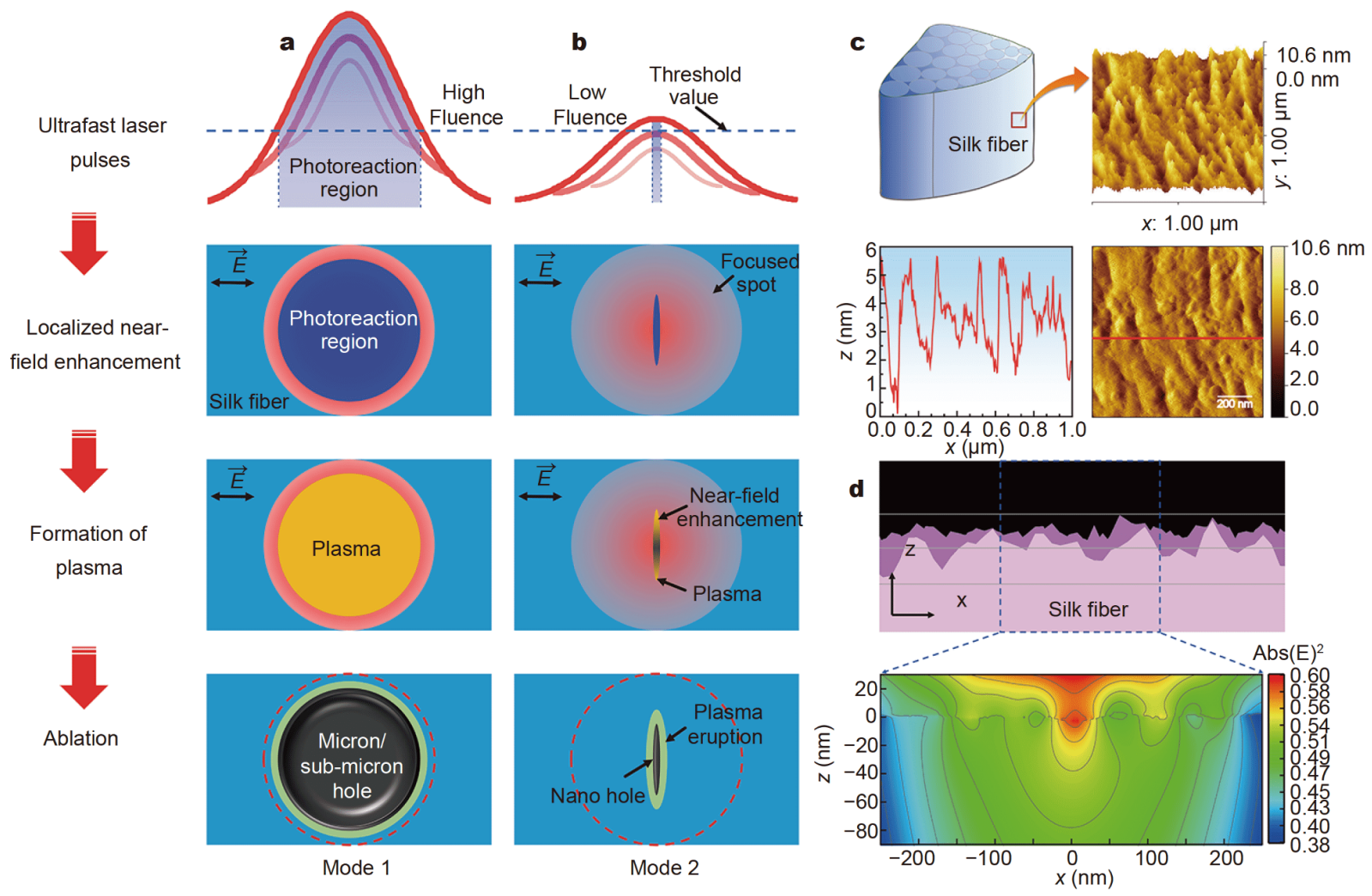

Figure 1 Two different modes for the processing of silk fiber using an ultrafast laser. (a) Schematic of Mode 1 for far-field laser ablation. (b) Schematic of Mode 2 for localized near-field enhancement-assisted ablation. (c) Surface morphology of a silk fiber. (d) A simulation model of the surface wavy structures and corresponding FDTD results of the optical intensity distribution.

lation of materials. The localized near-field enhancement effect is negligible with laser peak intensities much higher than the ablation threshold value (Fig. 1a). However, it dominates at the start of the ablation process when the laser peak intensity is just comparable to the ablation threshold value (Fig. 1b). FDTD method was used to analyze the optical intensity distribution in the near-field zone around the surface of the fiber. Fig. 1c shows the schematic and atomic force microscopy (AFM) images of the silk fiber. The surface of the fiber is covered with wavy structures with heights of about $10 \mathrm{~nm}$. Based on the morphology of the fiber, a simulation model was constructed and the optical intensity distribution was simulated (Fig. 1d). An increase of the optical intensity was observed in the nanocraters on the fiber surface. Optical intensity distributions near the fibers with different structures were calculated to confirm the effects of these structures on the near-field enhancement effect (Figs S2, S3). The cylindrical shape and a surface bulge show negative effects for the localized enhancement of optical intensity. Nanocraters with smaller radius and located near the center of the laser spot are favorable for the localized enhancement of optical intensity. Therefore, the realization of localized near-field enhancement-assisted ablation (Mode 2) is attributed to the nanostructures on the surface of the silk fiber. The localized near-field enhancement-assisted ablation procedure may also be used for nano-processing of synthetic materials with similar surface structures.

\section{Drilling on individual silk fiber using the femtosecond laser}

We demonstrated the processing strategies of the conventional far-field laser ablation (Mode 1) and our localized near-field enhancement-assisted ablation (Mode 2) by shooting individual silk fiber with 500 femtosecond laser pulses with pulse energy fluences varying from 3.58 to $8.06 \mathrm{~J} \mathrm{~cm}^{-2}$ (Fig. 2a and Fig. S4). There was a threshold of air breakdown, which was demonstrated to be about $9.5 \mathrm{~J} \mathrm{~cm}^{-2}$ for the laser used in this study [31]. The defocusing effects associated with air breakdown were not considered in this work. As shown in SEM images 

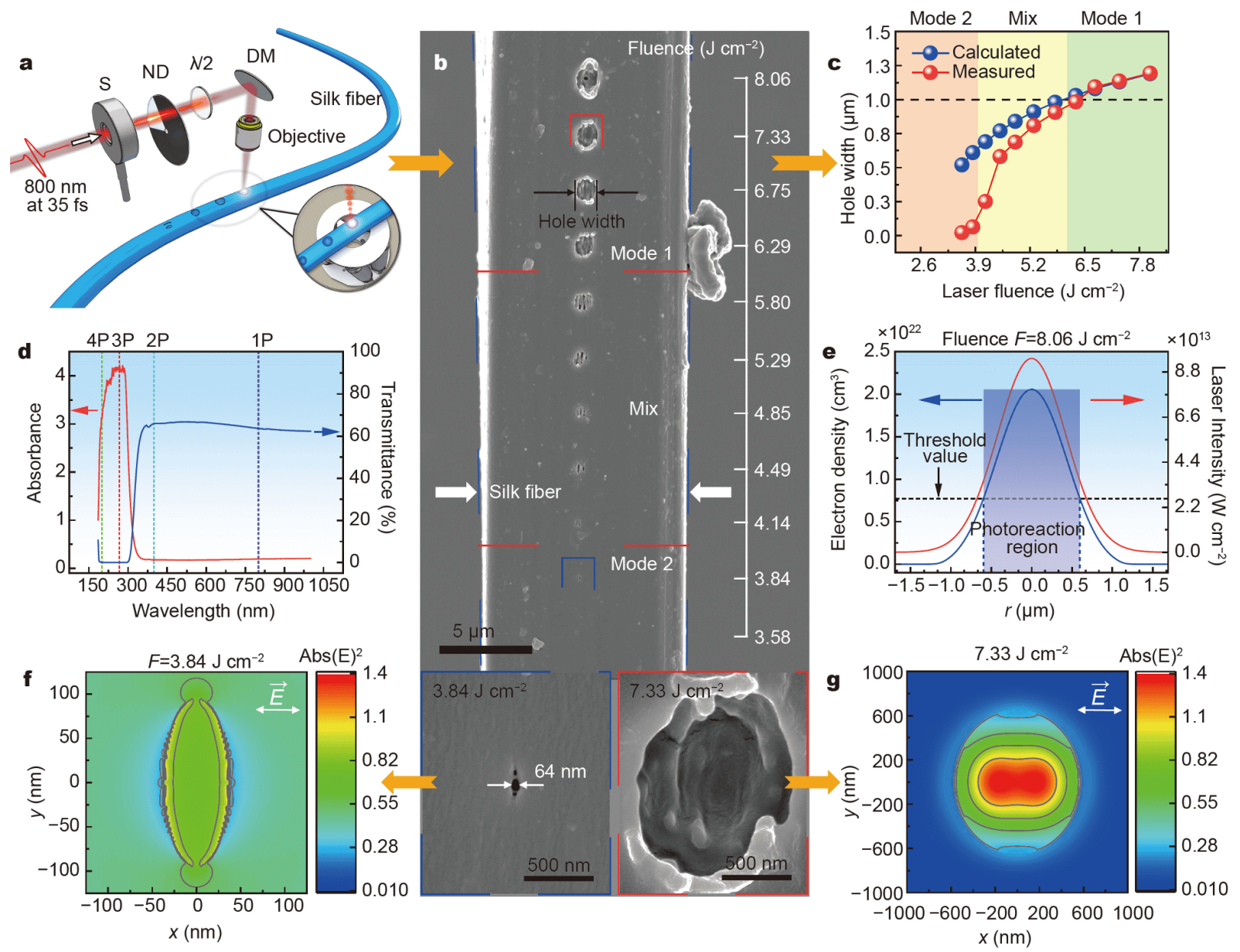

Figure 2 Controlled hole drilling on individual silk fiber by femtosecond laser pulses with different pulse energy fluences. (a) Schematic diagram of drilling holes on a silk fiber using a femtosecond laser. (b) SEM images of the holes drilled by 500 femtosecond laser pulses with different energy fluences. (c) The measured and calculated widths of the holes ablated with different pulse energy fluences. (d) Ultraviolet-visible-infrared absorbance and transmittance spectra of silk fibers in the range of 185-1,000 nm. (e) Spatial distribution of laser intensity and corresponding free electron density. (f, g) Calculated optical intensity distributions when the laser was focused on the fiber with holes drilled by 3.84 and $7.33 \mathrm{~J} \mathrm{~cm}^{-2}$ femtosecond laser pulses, respectively.

(Fig. 2b), the width of the nanohole drilled by the femtosecond laser increases as the pulse energy fluence increases (see Fig. S5 for more details). Fig. $2 \mathrm{c}$ shows the relationship between the hole width and pulse energy fluence. Based on the morphology and size of the holes, it can be identified that Mode 1 is dominant during the interaction between the silk fiber and laser pulses with high energy fluences $\left(\geq 6.29 \mathrm{~J} \mathrm{~cm}^{-2}\right.$ for 500 pulses). Furthermore, Mode 2 gradually becomes dominant, as the pulse energy fluence decreases continuously, which can decrease the dimension of the ablation hole. An elliptical hole with a width of $\sim 64 \mathrm{~nm}$ was successfully fabricated using $3.84 \mathrm{~J} \mathrm{~cm}^{-2}$ laser pulses. A smaller crater was observed at the position irradiated by $3.58 \mathrm{~J} \mathrm{~cm}^{-2}$ laser pulses (Fig. S5j), indicating that the method may achieve a smaller feature size. In comparison to the existing ultrafast laser processing technologies for silk fibroin materials, the feature size by Mode 2 is the minimum $(64 \mathrm{~nm})$, which is close to the limit in non-vacuum environment by nanoimprinting $(50 \mathrm{~nm})$ (Table S1).

A plasma model was used to quantify the mechanism behind the experimental results above. Owing to the biological dielectric characteristics of silk fibers, nonlinear effects (multiphoton absorption and avalanche ionization) dominate the ablation of the silk fibers when irradiated by a strong laser field [32]. The number of photon required to excite a valence electron to the conduction band can be acquired by studying the absorbance and transmittance spectra of the silk fiber, as shown in Fig. 2d. On one hand, the silk fiber exhibits a semitransparent 
response at one- and two-photon transitions near the wavelengths of $400 \mathrm{~nm}$ (cyan) and $800 \mathrm{~nm}$ (purple), respectively (vertical lines in Fig. 2d). On the other hand, the spectrum of a silk fiber has about 24 times higher absorption at three-photon transition around the wavelength of $267 \mathrm{~nm}$ (red) compared with the absorption at the wavelength of $400 \mathrm{~nm}$ (vertical lines in Fig. 2d). This suggests that three-photon ionization dominates during the initiation of the ionization process [33]. Avalanche ionization would occur subsequently [32]. The timedependent density of the free electrons $N(t, r)$ is defined by the rate equation [33-37]:

$\frac{\partial N(t, r)}{\partial t}=\omega_{\mathrm{mpi}} N_{\mathrm{a}}+\omega_{\mathrm{imp}} N(t, r)-\frac{N(t, r)}{\tau}$,

where $t$ is the time; $r$ is the distance to the beam axis; $\omega_{\mathrm{mpi}}$ is the probability for three-photon ionization; $N_{\mathrm{a}}$ is the density of neutral atoms and is $4.12 \times 10^{22} \mathrm{~cm}^{-3}$ for the silk fiber; $\omega_{\text {imp }}$ is the probability for avalanche ionization and $\tau \approx 1$ ps is the decay time constant [38]; $\omega_{\text {mpi }}$ and $\omega_{\text {imp }}$ are presented by

$\omega_{\mathrm{mpi}} \approx \omega n_{\mathrm{ph}}^{3 / 2}\left(\frac{\varepsilon_{\mathrm{OSC}}}{2 J_{\mathrm{i}}}\right)^{n_{\mathrm{ph}}}$,

$\omega_{\mathrm{imp}} \approx \frac{\varepsilon_{\mathrm{OSC}}}{J_{\mathrm{i}}}\left(\frac{2 \omega^{2} v_{\mathrm{eff}}}{\omega^{2}+v_{\mathrm{eff}}^{2}}\right)$,

where $\omega$ is the laser frequency; $\varepsilon_{\mathrm{OSC}}$ is the electron quiver energy in the ultrafast laser field; $n_{\mathrm{ph}}=3$ is the number of photons for multiphoton ionization; $J_{\mathrm{i}}=4 \mathrm{eV}$ is the ionization potential, and $v_{\text {eff }}$ is the effective collision frequency. The electron quiver energy can be expressed by

$\varepsilon_{\mathrm{OSC}}[\mathrm{eV}]=9.3 \times \frac{I(t, r)}{10^{14}\left[\mathrm{~W} / \mathrm{cm}^{3}\right]}(\lambda[\mu \mathrm{m}])^{2}$,

where $\lambda$ is the laser wavelength, and $I(t, r)$ is the laser intensity on the surface of the silk fiber. It can be expressed by

$I(t, r)=I_{0}(1-R(t, r)) \times \exp \left(-\frac{r^{2}}{r_{0}^{2}}-(4 \ln 2)\left(\frac{t}{t_{\mathrm{p}}}\right)\right)$,

where $I_{0}$ is the peak intensity and can be expressed by $I_{0}=2 F /\left(\sqrt{\pi / \ln 2 t_{\mathrm{p}}}\right) ; F$ is the pulse energy fluence; $t_{\mathrm{p}}$ is the pulse duration; $R(t, r)$ is the time-dependent reflectivity of the silk fiber, which can be described with the Drude model; $r_{0}$ is the beam radius defined by $I\left(r_{0}\right)=I_{0} \cdot e^{-2}$, and it is $0.845 \mu \mathrm{m}$ when a $50 \times$ microscope objective (NA = $0.5)$ is used (Fig. S6).

We calculated the dynamics of laser intensity, free electron density, reflectivity, and spatial distribution of maximum free electron density by the plasma model (Fig. S7). Ablation occurs when the free electron density is higher than the threshold value (Fig. 2e). Owing to the incubation effects [39], the threshold value decreases with increasing laser pulse number, and can be estimated by the free electron density near the edge of the micron-scale ablation holes. It is $7.7 \times 10^{21} \mathrm{~cm}^{-3}$ for 500 pulses and 5.3 $\times 10^{21} \mathrm{~cm}^{-3}$ for 1000 pulses in this study. The widths of the holes were estimated by comparing the maximum free electron density with the threshold value. The calculated widths of the holes are shown in Fig. 2c. The calculated hole widths are in good agreement with the measured ones in the region of Mode 1 (Fig. 2c and Fig. S8). However, with a decrease in the pulse energy fluence, the hole width cannot be estimated by the plasma model for the nonnegligible localized near-field enhancement (Fig. 1b)

To analyze the localized near-field enhancementassisted ablation by the femtosecond laser pulse train, the optical intensity distributions in the near-field zone of a circular nanocrater was calculated (Fig. S9). The optical intensity was redistributed. The enhanced intensity at the edges would lead to the ablation of the nanocrater along the direction perpendicular to the laser polarization. Thus an elliptical nanohole was processed and the optical intensity was concentrated inside the elliptical nanohole (Fig. $2 \mathrm{f}$ and Movie S1). In contrast, the local field enhancement was negligible when the dimension of the hole was larger than the laser wavelength (Fig. $2 \mathrm{~g}$ and Movie S2).

Furthermore, the localized micro- and nano-grooves with controlled widths can be easily processed by moving the focused spot (Figs S10, S11). For instance, the localized nanogrooves with controllable widths from $\sim 660$ to $\sim 80 \mathrm{~nm}$ and depths from $\sim 300$ to $\sim 70 \mathrm{~nm}$ were achieved by varying the pulse energy fluence from 5.27 to $2.64 \mathrm{~J} \mathrm{~cm}^{-2}$ and moving the focused spot at a speed of $1 \mu \mathrm{m} \mathrm{s}^{-1}$ (Fig. S10).

\section{Characterization of laser-processed silk fibers}

Non-invasive processing is essential to maintain the functions of native silk fibers. Specifically, the positions of the Raman peaks of the ablation sites are consistent with those of the native silk after femtosecond laser grooving, cutting, and side-cutting (Fig. 3a, Tables S2, S3). For example, the five Raman bands at $1084 \mathrm{~cm}^{-1}$ (attributed to the $\mathrm{C}-\mathrm{C}$ skeleton bond and $\mathrm{CH}_{3}$ vibrating) [40,41], $1230 \mathrm{~cm}^{-1}$ (attributed to amide III, N-H in-plane bending, and $\mathrm{CH}_{2}$ twisting) [40,41], $1446 \mathrm{~cm}^{-1}$ (attributed to $\mathrm{CH}_{3}$ anti-symmetric bending in polyalanine and $\mathrm{CH}_{2}$ bending in polyalanylglycine) $[40,42], 1664 \mathrm{~cm}^{-1}$ (attributed to amide $\mathrm{I}$ and $\mathrm{C}=\mathrm{O}$ stretching in $\beta$-sheets) 

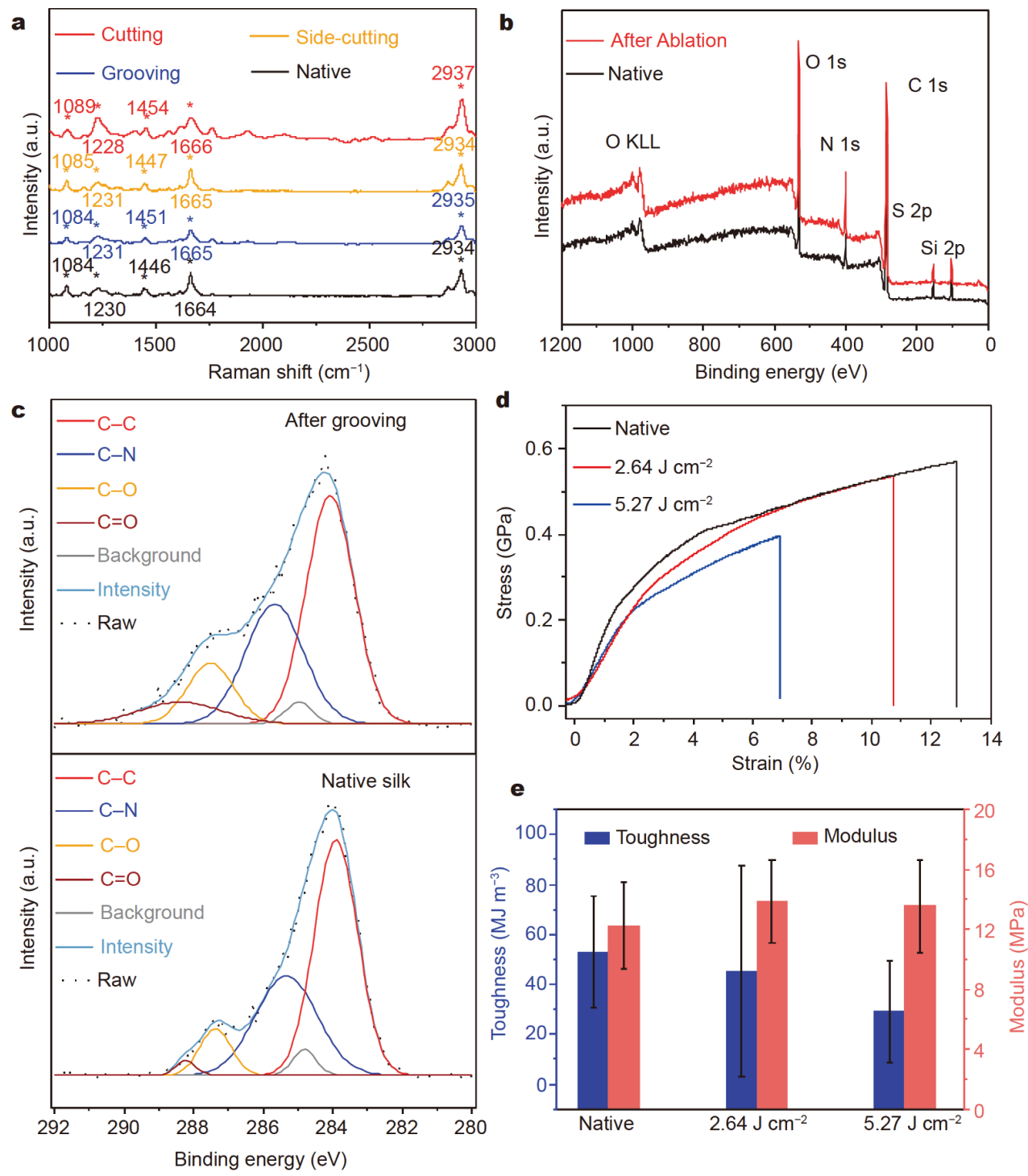

Figure 3 Characterization of laser-processed silk fibers. (a) Raman spectra of the silk fibers at different ablation sites and the spectrum of native silk fiber. (b) Survey XPS spectra of silk fabric before and after ablation with a femtosecond laser. The existing of a Si $2 p$ peak in the full XPS survey spectrum is attributed to a silicon wafer used as a substrate. (c) High-resolution XPS C1s spectrum of silk after ablation with a femtosecond laser (top) and the spectrum of native silkworm silk (bottom). (d) Stress-strain curves of degummed silk fibers after processing using femtosecond lasers with fluences of 2.64 and $5.27 \mathrm{~J} \mathrm{~cm}^{-2}$, along with that of the native silk. The stress was computed by assuming a constant silk diameter. (e) Comparison of the toughness and Young's modulus of processed silk and native silk. The error bars indicate standard deviations from seven different samples in each case.

$[40,43,44]$, and $2934 \mathrm{~cm}^{-1}$ (attributed to $\mathrm{CH}_{2}$ stretching) exhibit negligible shifts (not exceeding $\pm 8 \mathrm{~cm}^{-1}$ ) [45]. The shifts of the peaks may mainly be due to large variations caused by the natural variability of the silk sample. The preservation of most of the remarkable Raman bands after ablation suggests that the femtosecond laser is capable of non-invasive processing of silk fibers.

As shown in the spectra of XPS, the peak positions of various elements such as $\mathrm{C} 1 \mathrm{~s}, \mathrm{O} 1 \mathrm{~s}, \mathrm{~N} 1 \mathrm{~s}$, and $\mathrm{S} 2 \mathrm{p}$ that exist in the native silk exhibit negligible shifts before and after femtosecond laser ablation (Fig. 3 b and Table S4). In particular, the peak positions of the $\mathrm{C}-\mathrm{C}, \mathrm{C}-\mathrm{N}, \mathrm{C}-\mathrm{O}$, and $\mathrm{C}=\mathrm{O}$ bonds of the native silk are 283.9, 285.3, 287.4, and $288.2 \mathrm{eV}$ (Fig. 3c), respectively. In the ablation region, the peak positions of the $\mathrm{C}-\mathrm{C}, \mathrm{C}-\mathrm{N}, \mathrm{C}-\mathrm{O}$, and $\mathrm{C}=\mathrm{O}$ bonds are $284.1,285.7,287.5$, and $288.4 \mathrm{eV}$, respectively. The same conclusion was obtained by analyzing the peaks of other elements such as $\mathrm{O} 1 \mathrm{~s}, \mathrm{~N} 1 \mathrm{~s}$, and S 2p (Fig. S12), indicating that non-invasive processing of silk fabric was realized using a femtosecond laser. A detailed description 
of different positions of the groove is also given to illustrate the non-invasive processing achieved by using the femtosecond laser (Fig. S13 and Table S5).

We measured the mechanical properties of the silk fibers that were drilled by femtosecond lasers with different fluences. Fig. $3 \mathrm{~d}$ shows the stress-strain curves of the native silk and the degummed silk fibers drilled by femtosecond lasers with fluences of 2.64 and $5.27 \mathrm{~J} \mathrm{~cm}^{-2}$. The strength of the silk fiber drilled by a $2.64 \mathrm{~J} \mathrm{~cm}^{-2}$ femtosecond laser $(0.53 \mathrm{GPa})$ is of the same order of magnitude as the strength of the native silk fiber $(0.57 \mathrm{GPa}$, Fig. 3e). Compared with the mechanical strength of the silk fiber drilled by a $2.64 \mathrm{~J} \mathrm{~cm}^{-2}$ femtosecond laser, the lower mechanical strength of the silk fiber that drilled by a $5.27 \mathrm{~J} \mathrm{~cm}^{-2}$ one could be attributed to the bigger drilled holes. Furthermore, the modulus, toughness, and elongation at break of the silk fiber after processing using the femtosecond laser are comparable to or even higher than those of native silk fibers (Fig. 3e, Fig. S14, and Tables S69). This suggests that femtosecond laser processing reserves the excellent mechanical properties of the native silk fiber.

In contrast, the processing of silk fibers using long pulse lasers including a Raman laser $(532 \mathrm{~nm})$, a $\mathrm{CO}_{2}$ laser $(10.6 \mu \mathrm{m})$, and a blue laser $(450 \mathrm{~nm})$ could lead to destruction of the silk structures (Figs S15-S21). There were sharp expansions of silk fibers after processing (Figs S15, S16, and S19). Moreover, obvious bulge and ablation of the silk fabric were generated after processing using a $\mathrm{CO}_{2}$ laser and blue laser (Figs S17 and S20). The Raman spectra of both bulging and ablation regions showed distinct alterations (Figs S15d, S18c, and S21c), indicating that the structures of the silk around the processing area were no longer composed of silk protein. Furthermore, the fracture surface was flat after being cut by a femtosecond laser (Fig. S22). In contrast, the section was rough and showed many serrated structures after being cut using a $\mathrm{CO}_{2}$ laser (Fig. S23). Femtosecond laser fabrication is an ultrafast process endowed with nonthermal processing and low collateral damage. Compared with the femtosecond laser, long pulse lasers will generate lots of heat during the process and cause structure and composition damage.

\section{Biocompatible carrier and patterning on silk fabric}

As a kind of natural biological protein, a silk fiber possesses excellent biocompatibility [46]. Based on the noninvasive processing approach mentioned above, we fabricated a biocompatible carrier of micro/nanoparticles and drugs. For the sake of presentation, microholes with diameters of $5 \mu \mathrm{m}$ were processed on the silk fiber using the femtosecond laser and PS spheres with diameters of $3 \mu \mathrm{m}$ were selected (Fig. $4 \mathrm{a}, \mathrm{b}$ ). Then, owing to the van der Waals force between the Ti tip and PS microsphere, the PS microsphere was successfully adhered to the tip of Ti probe (Movie S3). Finally, the PS microsphere was successfully inserted into the hole (Movie S4). The entire process was carried out under an optical microscope. As a demonstration of the method, it is still difficult and time consuming to fabricate the biocompatible carrier. In the future, the processing efficiency can be further improved, for example it may be improved by multi-beam laser. Furthermore, by applying nanoscale manipulation techniques, nano-scale particles, drugs and so on can be put into the processed holes, indicating that the silk fiber can serve as a carrier after non-invasive processing. Furthermore, silk protein can be sufficiently decomposed, without adverse effects $[10,17,47,48]$. It might have promise to be used in many frontier areas such as bioengineering and bioelectronics. For example, it is possible to be used for real-time observation the life state of a single bacteria or cell under the influence of drugs. To prevent particles in the holes fall off during vibration or deformation, silk solution could be dropped on the surface of the holes to fix and encapsulate the particles.

Besides, the logo of Tsinghua University with a dimension of $5.0 \mathrm{~mm} \times 1.7 \mathrm{~mm}$ was patterned on the silk fabrics to demonstrate the ability of the femtosecond laser fabrication system (Fig. 4c-f). Femtosecond laser pulses with a repetition frequency and fluence of $1000 \mathrm{~Hz}$ and $21.40 \mathrm{~J} \mathrm{~cm}^{-2}$, respectively, were focused on the surface of a silk fabric with a writing speed of $1 \mathrm{~mm} \mathrm{~s}^{-1}$. The gap of the ablation areas was set as $25 \mu \mathrm{m}$ to shorten the processing time. A coin of ten cents and a piece of fabric processed by the femtosecond laser, on which the logo of Tsinghua University was patterned, are shown in Fig. 4c. There are distinct patterns in optical microscopy images (Fig. $4 \mathrm{~d}-\mathrm{f}$ ) with different amplifications of the logo in Fig. 4c. Therefore, the femtosecond laser can not only process individual silk fiber, but also silk fabric to achieve aesthetic designs.

\section{CONCLUSIONS}

In conclusion, we reported a novel approach for nanoprocessing of individual silkworm silk fibers using an ultrafast laser with localized near-field enhancement. Two different modes, far-field laser ablation and near-field enhancement-assisted ablation were used for laser processing. We exploited a wide variety of non-invasive localized shaping capabilities such as nano-holing, nano- 

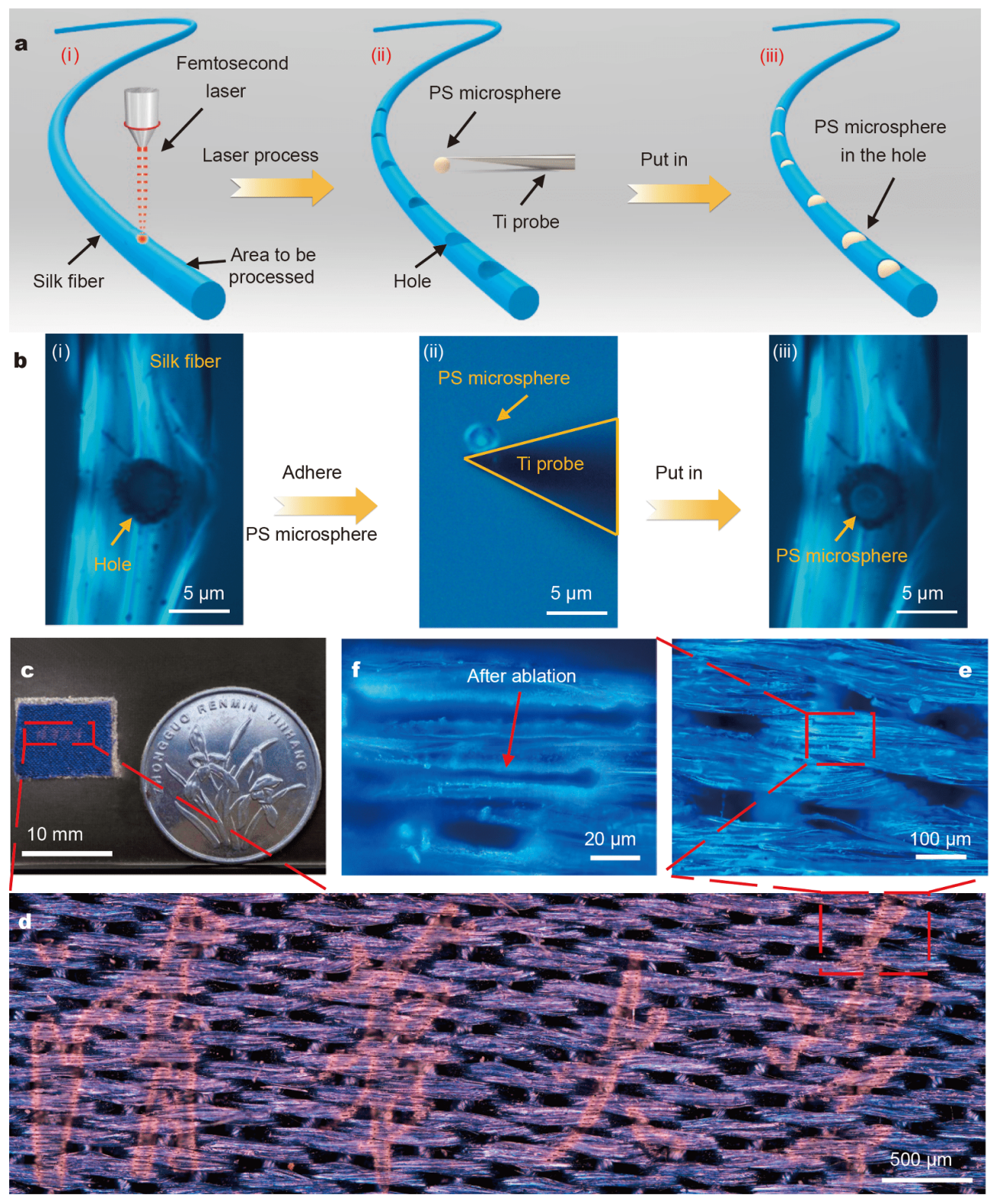

Figure 4 Biocompatible carrier and patterning on silk fabric. (a) A silk fiber as a biocompatible carrier after laser processing. Schematic diagram of the transfer of a PS microsphere into the hole on the silk fiber, (i) perforating individual silk fiber using the femtosecond laser, (ii) a PS microsphere at the tip of the probe, and (iii) a PS microsphere in the hole on the silk fiber. (b) Experimental optical images of the transfer of a PS microsphere into the hole on the silk fiber, (i) a hole on silk fiber, (ii) a PS microsphere at the tip of the probe, and (iii) a PS microsphere in the hole on the silk fiber. (c) The logo of Tsinghua University was patterned on to the silk fabric using the femtosecond laser. There is a coin of ten cents on the right of the silk fabric. (d) The optical microscopy image of the logo of Tsinghua University. (e, f) Optical microscopy images at different amplifications corresponding to the square area shown in (d) showing the ablation of the silk after processing with the femtosecond laser.

grooving, and cutting, with negligible structural damage. By carefully controlling the peak intensities to the threshold value, a narrow hole with size down to $\sim 64 \mathrm{~nm}$ was fabricated. The minimum depth and width of the nanogrooves were 70 and $80 \mathrm{~nm}$, respectively. Furthermore, the fibers retained the key molecular structure building blocks, the pristine functionality, and excellent mechanical properties after processing with the femtosecond laser. Our study has implications for a diverse range of applications. For example, the transfer of micro/ nanoparticle and drugs can be exploited for biomedical engineering. Furthermore, the femtosecond laser can not only process individual silk fibers, but also process fabric to achieve aesthetic designs. The processing strategy de- 
veloped in this study could also be extended to other materials, paving a new way for fabricating functional nanostructures with precisely controlled size and morphology.

\section{Received 29 February 2020; accepted 12 April 2020; published online 30 April 2020}

1 Omenetto FG, Kaplan DL. New opportunities for an ancient material. Science, 2010, 329: 528-531

2 Yan M, Ma X, Yang Y, et al. Biofabrication strategy for functional fabrics. Nano Lett, 2018, 18: 6017-6021

3 Jia T, Wang Y, Dou Y, et al. Moisture sensitive smart yarns and textiles from self-balanced silk fiber muscles. Adv Funct Mater, 2019, 29: 1808241

4 Zhang W, Ye C, Zheng K, et al. Tensan silk-inspired hierarchical fibers for smart textile applications. ACS Nano, 2018, 12: 69686977

5 Huby N, Vié V, Renault A, et al. Native spider silk as a biological optical fiber. Appl Phys Lett, 2013, 102: 123702

6 Tao H, Kaplan DL, Omenetto FG. Silk materials-a road to sustainable high technology. Adv Mater, 2012, 24: 2824-2837

7 Mondia JP, Amsden JJ, Lin D, et al. Rapid nanoimprinting of doped silk films for enhanced fluorescent emission. Adv Mater, 2010, 22: 4596-4599

8 Kim S, Marelli B, Brenckle MA, et al. All-water-based electronbeam lithography using silk as a resist. Nat Nanotech, 2014, 9: 306310

9 Sun YL, Li Q, Sun SM, et al. Aqueous multiphoton lithography with multifunctional silk-centred bio-resists. Nat Commun, 2015, 6: 8612

10 Hwang SW, Tao H, Kim DH, et al. A physically transient form of silicon electronics. Science, 2012, 337: 1640-1644

11 Capelli R, Amsden JJ, Generali G, et al. Integration of silk protein in organic and light-emitting transistors. Org Electron, 2011, 12: 1146-1151

12 Tao H, Hwang SW, Marelli B, et al. Silk-based resorbable electronic devices for remotely controlled therapy and in vivo infection abatement. Proc Natl Acad Sci USA, 2014, 111: 17385-17389

13 Kim T, McCall JG, Jung YH, et al. Injectable, cellular-scale optoelectronics with applications for wireless optogenetics. Science, 2013, 340: 211-216

14 Barr MC, Rowehl JA, Lunt RR, et al. Direct monolithic integration of organic photovoltaic circuits on unmodified paper. Adv Mater, 2011, 23: 3500-3505

15 Hübler A, Trnovec B, Zillger T, et al. Printed paper photovoltaic cells. Adv Energy Mater, 2011, 1: 1018-1022

16 Tang-Schomer MD, Hu X, Hronik-Tupaj M, et al. Film-based implants for supporting neuron-electrode integrated interfaces for the brain. Adv Funct Mater, 2014, 24: 1938-1948

17 Kim DH, Viventi J, Amsden JJ, et al. Dissolvable films of silk fibroin for ultrathin conformal bio-integrated electronics. Nat Mater, 2010, 9: 511-517

18 Maximova K, Wang X, Balčytis A, et al. Silk patterns made by direct femtosecond laser writing. Biomicrofluidics, 2016, 10: 054101

19 Moore A, Koch M, Mueller K, et al. Precise laser ablation processing of black widow spider silk. Appl Phys A, 2003, 77: 353-357
20 Jiang HB, Zhang YL, Han DD, et al. Bioinspired fabrication of superhydrophobic graphene films by two-beam laser interference. Adv Funct Mater, 2014, 24: 4595-4602

21 Tirlapur UK, König K. Targeted transfection by femtosecond laser. Nature, 2002, 418: 290-291

22 Hanczyc P, Samoc M, Norden B. Multiphoton absorption in amyloid protein fibres. Nat Photon, 2013, 7: 969-972

23 Zhang YL, Chen QD, Xia H, et al. Designable 3D nanofabrication by femtosecond laser direct writing. Nano Today, 2010, 5: 435-448

24 Sidhu MS, Kumar B, Singh KP. The processing and heterostructuring of silk with light. Nat Mater, 2017, 16: 938-945

$25 \mathrm{Xu} \mathrm{BB}$, Xia H, Niu LG, et al. Flexible nanowiring of metal on nonplanar substrates by femtosecond-laser-induced electroless plating. Small, 2010, 6: 1762-1766

26 Wang S, Yu Y, Ma D, et al. High performance hybrid supercapacitors on flexible polyimide sheets using femtosecond laser 3D writing. J Laser Appl, 2017, 29: 022203

27 Tanaka Y, Obara M. Comparison of resonant plasmon polaritons with MIE scattering for laser-induced near-field nanopatterning: Metallic particle $v s$ dielectric particle. Jpn J Appl Phys, 2009, 48: 122002

28 Miyanishi T, Sakai T, Nedyalkov NN, et al. Femtosecond-laser nanofabrication onto silicon surface with near-field localization generated by plasmon polaritons in gold nanoparticles with oblique irradiation. Appl Phys A, 2009, 96: 843-850

29 Lee JY, Hong BH, Kim WY, et al. Near-field focusing and magnification through self-assembled nanoscale spherical lenses. Nature, 2009, 460: 498-501

30 Tanaka Y, Obara G, Zenidaka A, et al. Femtosecond laser nearfield nanoablation patterning using MIE resonance high dielectric constant particle with small size parameter. Appl Phys Lett, 2010, 96: 261103

31 Xu S, Yao C, Liao W, et al. Experimental study on $800 \mathrm{~nm}$ femtosecond laser ablation of fused silica in air and vacuum. Nucl Instruments Methods Phys Res Sect B-Beam Interactions Mater Atoms, 2016, 385: 46-50

32 Balling P, Schou J. Femtosecond-laser ablation dynamics of dielectrics: Basics and applications for thin films. Rep Prog Phys, 2013, 76: 036502

33 Xiong W, Zhou YS, He XN, et al. Simultaneous additive and subtractive three-dimensional nanofabrication using integrated two-photon polymerization and multiphoton ablation. Light Sci Appl, 2012, 1: e6

34 Stuart BC, Feit MD, Herman S, et al. Nanosecond-to-femtosecond laser-induced breakdown in dielectrics. Phys Rev B, 1996, 53: 1749-1761

35 Jiang L, Wang AD, Li B, et al. Electrons dynamics control by shaping femtosecond laser pulses in micro/nanofabrication: Modeling, method, measurement and application. Light Sci Appl, 2018, 7: 17134

36 Qiao M, Yan J, Gao B. Ablation of $\mathrm{TiO}_{2}$ surface with a doublepulse femtosecond laser. Optics Commun, 2019, 441: 49-54

37 Gamaly EG, Rode AV, Luther-Davies B, et al. Ablation of solids by femtosecond lasers: Ablation mechanism and ablation thresholds for metals and dielectrics. Phys Plasmas, 2002, 9: 949-957

38 Lazare S, Sionkowska A, Zaborowicz M, et al. Bombyx mori silk protein films microprocessing with a nanosecond ultraviolet laser and a femtosecond laser workstation: Theory and experiments. Appl Phys A, 2012, 106: 67-77

39 Mero M. On the damage behavior of dielectric films when illu- 
minated with multiple femtosecond laser pulses. Opt Eng, 2005, 44: 051107

40 Moore WH, Krimm S. Vibrational analysis of peptides, polypeptides, and proteins. II. $\beta$-Poly(L-alanine) and $\beta$-poly(L-alanylglycine). Biopolymers, 1976, 15: 2465-2483

41 Rousseau ME, Lefèvre T, Beaulieu L, et al. Study of protein conformation and orientation in silkworm and spider silk fibers using Raman microspectroscopy. Biomacromolecules, 2004, 5: 22472257

42 Krimm S, Banderkar J. Vibrational spectroscopy and conformation of peptides, polypeptides, and proteins. Adv Protein Chem, 1986, 38: $181-364$

43 Krimm S. Vibrational analysis of conformation in peptides, polypeptides, and proteins. Biopolymers, 1983, 22: 217-225

44 Moore WH, Krimm S. Vibrational analysis of peptides, polypeptides, and proteins. I. Polyglycine i. Biopolymers, 1976, 15: 2439-2464

45 Qiu W, Teng W, Cappello J, et al. Wet-spinning of recombinant silk-elastin-like protein polymer fibers with high tensile strength and high deformability. Biomacromolecules, 2009, 10: 602-608

46 Porter D, Vollrath F. Silk as a biomimetic ideal for structural polymers. Adv Mater, 2009, 21: 487-492

47 Mannoor MS, Tao H, Clayton JD, et al. Graphene-based wireless bacteria detection on tooth enamel. Nat Commun, 2012, 3: 763

48 Qin Z, Compton BG, Lewis JA, et al. Structural optimization of 3D-printed synthetic spider webs for high strength. Nat Commun, 2015, 6: 7038

Acknowledgements We thank Maosheng Chai, Shuang Li, and Dr. Han Li for the help with experimental tests. We thank Dr. Baihui Liang, Dr. Taotao Sun, Dr. Hengqian Hu, and Dr. Chuang Li for the experimental assistance and discussions. We acknowledge the support from the National Key R\&D Program of China (2017YFB1104300, 2016YFA0200103 and 2018YFB1107200), the National Program for the Support of Top-notch Young Professionals, and the National Natural Science Foundation of China (51775303).

Author contributions Qiao $\mathrm{M}$ and Wang $\mathrm{H}$ contributed equally to this work. Yan J and Zhang Y supervised the project. Qiao M contributed to the nano-processing of silk fibers, mechanism analysis, and simulations. Wang $\mathrm{H}$ and Qiao $\mathrm{M}$ conducted the characterization of SEM. Wang $\mathrm{H}$ contributed to the AFM characterization, Raman measurements, XPS analysis, and microparticle transfer experiment. Lu $\mathrm{H}$ performed the mechanical measurements. Li S prepared the degummed silk fibers. Qu L, Jiang L and Lu Y guided the theoretical analysis part. Wang H, Qiao M, Yan J and Zhang Y co-wrote the paper with feedback from all authors.

Conflict of interest The authors declare no conflict of interest.

Supplementary information Experimental details and supporting data are available in the online version of the paper.

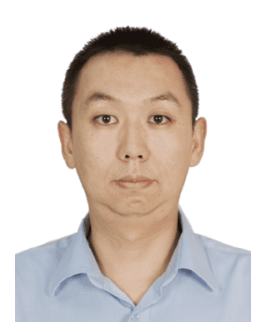

Ming Qiao received his master's degree in mechanical engineering from the University of Chinese Academy of Sciences in 2015. He is currently a $\mathrm{PhD}$ candidate at the Department of Mechanical Engineering, Tsinghua University. His research interests focus on femtosecond laser and micro/nano fabrication.

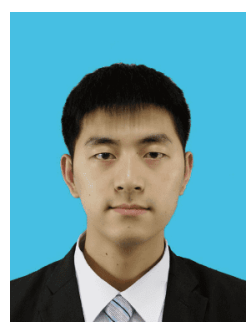

Huimin Wang received his BSc degree in inorganic nonmetallic material engineering from Jilin University in 2016. He is currently a $\mathrm{PhD}$ candidate at the Department of Chemistry, Tsinghua University, China. His current research interest focuses on nanocarbon, silk, and their hybrid materials and their applications in flexible electronics.

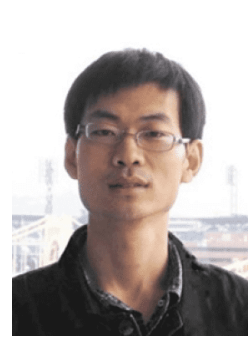

Jianfeng Yan received his $\mathrm{PhD}$ from the Department of Mechanical Engineering, Tsinghua University in 2013. He is currently an associate professor, Department of Mechanical Engineering, Tsinghua University. His research interests are mainly focused on ultrafast laser and micro/ nano fabrication.

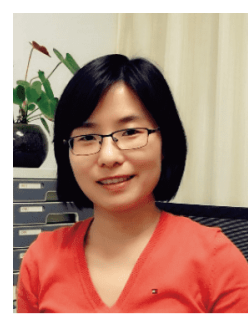

Yingying Zhang received her $\mathrm{PhD}$ degree in physical chemistry from Peking University in 2007. From June 2008 to June 2011, she worked at Los Alamos National Laboratory (USA) as a postdoctoral research associate. Then, she joined Tsinghua University as an associate professor in July of 2011. Her research focuses on the design and controlled preparation of nanocarbon, silk, and their hybrid materials, aiming to develop high-performance flexible electronics and wearable systems.

\section{利用近场增强超快激光对天然蚕丝进行微纳加工}

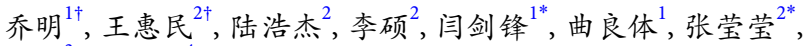
姜澜 ${ }^{3}$, 陆永枫 $^{4}$

摘要 桑虫丝因具有突出的力学性能、优异的光学特性和良好的 生物相容性而备受关注. 但目前尚缺乏对单根天然虫丝进行纳米 级加工的手段, 因而限制了虫丝在纳米工程等领域的应用. 本文利 用局部近场增强超快激光在虫丝表面实现了纳米级加工, 并进一 步实现了纳米孔、纳米槽的制备以及对虫丝的精密切割. 形貌和 成分测试结果表明, 该方法在不破坏虫丝关键分子结构模块和原 有功能的前提下实现了最小宽度仅为 $64 \mathrm{~nm}$ 椭圆孔的加工. 此外, 本文还将超快激光加工的亘丝用于微纳颗粒、药物输运, 预示了 本方法在生物医学工程领域的广泛应用前景. 本文所报道的近场 增强超快激光加工方法不仅可用于虫丝的纳米级加工，也有望在 其他材料上实现高精度、可控制备功能性纳米结构. 